\title{
A METHOD FOR SEPARABLE NONLINEAR LEAST SQUARES PROBLEMS WITH SEPARABLE NONLINEAR EQUALITY CONSTRAINTS*
}

\author{
LINDA KAUFMAN† AND VICTOR PEREYRA
}

\begin{abstract}
Recently several algorithms have been proposed for solving separable nonlinear least squares problems which use the explicit coupling between the linear and nonlinear variables to define a new nonlinear least squares problem in the nonlinear variables only whose solution is the solution to the original problem. In this paper we extend these techniques to the separable nonlinear least squares problem subject to separable nonlinear equality constraints.
\end{abstract}

1. Introduction. In this paper we will consider the nonlinear least squares problem of finding $\mathbf{a}$ and $\boldsymbol{\alpha}$ which minimize

$$
\|\mathbf{y}-\Phi(\boldsymbol{\alpha}) \mathbf{a}\|_{2}^{2}
$$

subject to nonlinear equality constraints of the form

$$
H(\boldsymbol{\alpha}) \mathbf{a}=\mathbf{g}(\boldsymbol{\alpha}) .
$$

The abbreviated notation of (1.1) has the following meaning:

$$
\begin{array}{lr}
\Phi_{i j}(\boldsymbol{\alpha})=\left(\phi_{j}\left(\boldsymbol{\alpha} ; t_{i}\right)\right), & i=1, \cdots, m, \quad j=1, \cdots, n, \\
\mathbf{a}=\left(a_{1}, \cdots, a_{n}\right)^{T}, \quad \mathbf{y}=\left(y_{1}, \cdots, y_{m}\right)^{T}, \quad \boldsymbol{\alpha}=\left(\alpha_{1}, \cdots, \alpha_{k}\right)^{T} .
\end{array}
$$

In (1.2) we have $p$ nonlinear constraints, i.e. $\mathbf{g}(\boldsymbol{\alpha})=\left(g_{1}(\boldsymbol{\alpha}), \cdots, g_{p}(\boldsymbol{\alpha})\right)^{T}$, and $H(\boldsymbol{\alpha})$ is a $p \times n$ nonlinear matrix function $(p \leqq n+k)$. All the functions involved are assumed to be at least twice continuously differentiable, though somewhat weaker hypotheses could be employed.

In [2], Golub and Pereyra have discussed unconstrained problems of the form (1.1) which they have denoted as "separable nonlinear least squares problems".

Krogh [7] has extended those results to the more general models

$$
\|\mathbf{y}-\boldsymbol{\Psi}(\boldsymbol{\alpha})-\Phi(\boldsymbol{\alpha}) \mathbf{a}\|_{2}^{2}
$$

We do not need to introduce $\boldsymbol{\Psi}(\boldsymbol{\alpha})$ explicitly in our present formulation, since it can be included in $\Phi(\boldsymbol{\alpha}) \mathbf{a}$ as $a_{n+1} \Psi(\boldsymbol{\alpha})$ provided we add the constraint $a_{n+1}=1$. In [5] one of the authors has introduced more substantial modifications which simplify even further the algorithm.

Constraints of the form (1.2) appear in the applications [6] and they are considered here because of their similarity with (1.1). These problems can be reduced to unconstrained separable problems with a somewhat more complex structure. This is developed in detail in $\S 2$. Once the reduction is performed, one could use any program available for unconstrained separable problems. However, it turns out to be considerably more efficient to devise a completely new algorithm,

\footnotetext{
* Received by the editors October 31, 1975, and in revised form August, 1976.

$\dagger$ Department of Computer Science, University of Colorado, Boulder, Colorado. Now at Bell Laboratories, Murray Hill, New Jersey 07974.

$\ddagger$ Department of Mathematics, Universidad Central de Venezuela, Caracas, Venezuela, and Department of Mathematics, California Institute of Technology, Pasadena, California 91125.
} 
taking into account the structure of the problem, as we have done in $\S 3$ of this paper.

It has been shown in practice [2], [7] and there are some theoretical indications [10], that the separation of the linear variables a from the nonlinear variables $\boldsymbol{\alpha}$ by means of the variable projection method [2], [3], [5], [8] speeds up the convergence of iterative methods used to solve problem (1.1).

We extend in this paper the range of applicability of the variable projection method to constrained problems.

The reduction to an unconstrained problem at the beginning of $\S 2$ was anticipated in [3].

2. The reduction to an unconstrained separable problem. In this section we consider the problem of finding vectors $\hat{\mathbf{a}}$ and $\hat{\boldsymbol{\alpha}}$ which minimize

$$
r(\mathbf{a}, \boldsymbol{\alpha})=\|\mathbf{y}-\Phi(\boldsymbol{\alpha}) \mathbf{a}\|_{2}^{2}
$$

subject to the nonlinear equality constraints

$$
H(\boldsymbol{\alpha}) \mathbf{a}=\mathbf{g}(\boldsymbol{\alpha}),
$$

where all the vectors and matrices are as in $\S 1$. In what follows, an upper superscript ${ }^{+}$on a matrix will denote its Moore-Penrose generalized inverse (see [9]).

In order to guarantee the existence of feasible points we assume that there are vectors $\boldsymbol{\alpha}$ for which the resulting linear systems $(2.1 b)$ is compatible; i.e. $\mathbf{g}(\boldsymbol{\alpha}) \in$ range $(H(\alpha))$, or equivalently, $\mathbf{g}(\boldsymbol{\alpha})=H(\boldsymbol{\alpha}) H^{+}(\boldsymbol{\alpha}) \mathbf{g}(\boldsymbol{\alpha})$. The set of all such vectors $\alpha$ will be denoted by $A$.

For each fixed $\alpha \in A$, the general solution of the resulting system of linear equations $(2.1 \mathrm{~b})$ is given by [8]

$$
\mathbf{a}=H^{+}(\boldsymbol{\alpha}) \mathbf{g}(\boldsymbol{\alpha})+Y(\boldsymbol{\alpha}) \mathbf{z},
$$

with $H^{+}(\boldsymbol{\alpha})^{T} Y(\alpha)=0$, and $\mathrm{z}$ varying over all $R^{n-r}$ where $r$ is the rank of $H$. In other words, the columns of $Y(\alpha)$ are a basis for the null space of $H(\alpha)$. The set of all pairs $(\mathbf{a}, \boldsymbol{\alpha})$, where $\boldsymbol{\alpha} \in A$, and $\mathbf{a}$ is defined in (2.2), is the feasible set for problem (2.1). Therefore, this problem is equivalent to minimizing in $\mathbf{z}$ and $\boldsymbol{\alpha}$,

$$
\begin{aligned}
s(\mathbf{z}, \boldsymbol{\alpha}) & =\left\|\mathbf{y}-\Phi(\boldsymbol{\alpha}) H(\boldsymbol{\alpha})^{+} \mathbf{g}(\boldsymbol{\alpha})-\Phi(\boldsymbol{\alpha}) Y(\boldsymbol{\alpha}) \mathbf{z}\right\|_{2}^{2} \\
& \equiv\|\mathbf{y}-\zeta(\boldsymbol{\alpha})-G(\boldsymbol{\alpha}) \mathbf{z}\|_{2}^{2} .
\end{aligned}
$$

where

$$
\zeta(\boldsymbol{\alpha})=\Phi(\boldsymbol{\alpha}) H(\boldsymbol{\alpha})^{+} \mathbf{g}(\boldsymbol{\alpha}) \text { and } \quad G(\boldsymbol{\alpha})=\Phi(\boldsymbol{\alpha}) Y(\boldsymbol{\alpha}) .
$$

If the dimension $(n-r)$ of $\mathbf{z}$ is nonzero, then we would have a separable problem of the form (1.3). Since we want to apply the variable projection technique we will assume that $r<n$.

This problem could then be solved with any program for unconstrained separable problems by simply giving it the appropriate information. Once $\mathbf{z}$ is computed in the standard fashion, $\mathbf{a}$ is found by an application of formula (2.2). However, we would like to develop a completely new and more efficient algorithm, avoiding all redundant computation. 
Let

$$
\mathbf{f}(\boldsymbol{\alpha})=V(\boldsymbol{\alpha})^{T}(\mathbf{y}-\zeta(\boldsymbol{\alpha}))
$$

where $V(\boldsymbol{\alpha})$ is an orthogonal basis for the null space of $G(\boldsymbol{\alpha})$. Using a proof similar to Theorem 2.1 of [2], one can show that an $\boldsymbol{\alpha}$ which minimizes $t(\boldsymbol{\alpha})=\|\mathbf{f}(\boldsymbol{\alpha})\|_{2}^{2}$ also minimizes $s(\mathbf{z}, \boldsymbol{\alpha})$. Except for the terms involving $\zeta(\boldsymbol{\alpha})$, the function $t(\boldsymbol{\alpha})$ is similar to $r_{3}(\boldsymbol{\alpha})$ of equation (4.1) in Kaufman [5]. The Marquardt-Levenberg algorithm applied to $t(\boldsymbol{\alpha})$ using the derivative formula for $r_{3}(\boldsymbol{\alpha})$ in [3] modified to account for $\zeta(\boldsymbol{\alpha})$, gives the following scheme for generating the required $\alpha$ : one starts with an arbitrary $\boldsymbol{\alpha}^{(0)}$ and, until convergence is attained, generates the vectors $\boldsymbol{\alpha}^{(j)}$ by the rule

$$
\boldsymbol{\alpha}^{(j+1)}=\boldsymbol{\alpha}^{(j)}-\left(\frac{B}{\nu_{j} I}\right)^{+}\left(\frac{\mathbf{f}\left(\boldsymbol{\alpha}^{(j)}\right)}{\mathbf{0}}\right)_{\} k},
$$

where $\nu_{j}$ is large enough so that

$$
\left\|\mathbf{f}\left(\boldsymbol{\alpha}^{(j+1)}\right)\right\|_{2} \leqq\left\|\mathbf{f}\left(\boldsymbol{\alpha}^{(j)}\right)\right\|_{2}
$$

and $B=V^{T}\left[-D(\zeta)-D(G) G^{-}(\mathbf{y}-\zeta)\right]$. The operator $D$ represents the Fréchet derivative with respect to $\alpha$, and $G^{-}$is any matrix satisfying $G G^{-} G=G$ and $\left(G G^{-}\right)^{T}=G G^{-}$.

Once $B$ and $\mathbf{f}\left(\boldsymbol{\alpha}^{(j)}\right)$ have been computed, one may efficiently obtain trial values of $\boldsymbol{\alpha}^{(j+1)}$ for various values of $\nu_{j}$ using the algorithm of [2].

A vector $\mathbf{z}$ which minimizes $s(\mathbf{z}, \boldsymbol{\alpha})$ for fixed $\boldsymbol{\alpha}$ is then given by $G^{-}(\boldsymbol{\alpha})(\mathbf{y}-\zeta(\boldsymbol{\alpha}))$.

The compact formula for $B$ in (2.5) is in terms of $D(\zeta)$ and $D(G)$. A more convenient expression for $B$ for the implementation of the algorithm is given in the following theorem:

THEOREM.

$$
B=-V^{T}\left\{\Phi H^{+}\left[-D(H) \mathbf{b}+H^{+\mathbf{T}} D\left(H^{T}\right) P_{H}^{\perp} \mathbf{g}+D(\mathbf{g})\right]+D(\Phi) \mathbf{b}\right\}
$$

where $\mathbf{b}=Y G^{-1}(\mathbf{y}-\zeta)+H^{+} \mathbf{g}$.

Proof. By (2.3),

$$
D(G)=\Phi D(Y)+D(\Phi) Y
$$

and

$$
D(\zeta)=D(\Phi) H^{+} \mathbf{g}+\Phi D\left(H^{+}\right) \mathbf{g}+\Phi H^{+} D(\mathbf{g}) .
$$

Thus, to obtain an expression for $B$, we need expressions for $D\left(H^{+}\right)$and $D(Y)$.

Golub and Pereyra [2] have proved that

$$
D\left(H^{+}\right)=-H^{+} D(H) H^{+}+H^{+} H^{+T} D(H)^{T} P_{H}^{\perp}+{ }_{H} P^{\perp} D(H)^{T} H^{+T} H^{T}
$$

where

$$
P_{H}^{\perp}=I-H H^{+}
$$

and

$$
{ }_{H} P^{\perp}=I-H^{+} H=Y Y^{T}
$$


When this formula is inserted into (2.8) and then into (2.5), the last term of (2.9) is canceled since

$$
V^{T} \Phi_{H} P^{\perp}=V^{T} \Phi Y Y^{T}=V^{T} G Y^{T}=0 .
$$

To obtain a formula for $D(Y)$ we'll use the orthogonal decomposition of $H$ given by

$$
H=Q^{T}\left(\begin{array}{l|l}
T & 0 \\
\hline 0 & 0
\end{array}\right) Z^{T}
$$

where $Q$ and $Z$ are orthogonal matrices and $T$ is an $r \times r$ nonsingular upper triangular matrix where $H$ has rank $r$. It is easy to verify that

$$
H^{+}=Z\left(\begin{array}{l|l}
T^{-1} & 0 \\
\hline 0 & 0
\end{array}\right) Q
$$

and that if $Z$ is partitioned as

$$
Z^{T}=\left[-\frac{Z_{1}^{T}}{Z_{2}^{T}}\right]_{\} n-r}^{\} r}
$$

then $H^{+T} Z_{2}=0$. Thus one may set $Y=Z_{2}$. we have

A formula for $D(Y)$ can be derived using the ideas of $\S 4$ of [5]. From (2.10)

$$
Z^{T} H^{T} Q^{T}=\left(\begin{array}{l|l}
T^{T} & 0 \\
\hline 0 & 0
\end{array}\right)
$$

which, according to $\S 4$ of [5], implies that there exists a matrix $M$ such that

$$
\frac{\partial Z_{2}^{T}}{\partial \alpha_{i}}=-Z_{2}^{T} \frac{\partial H^{T}}{\partial \alpha_{i}} H^{T+}+M Z_{2}^{T} .
$$

This means that

$$
D(Y)=-H^{+} D(H) Z_{2}+Z_{2} M^{T} .
$$

The matrix $Z_{2}$ is not unique and $M$ depends on which $Z_{2}$ is computed. Fortunately, when (2.11) is inserted into (2.7) and then into (2.5) the term with $Z_{2} M^{T}$ is canceled since

$$
V^{T} \Phi Z_{2} M^{T}=V^{T} \Phi Y M^{T}=V^{T} G M^{T}=0 .
$$

Thus one does not have to be concerned about $M$.

Combining (2.5), (2.7), (2.8), (2.9) and (2.10) we have

$$
\begin{aligned}
B=-V^{T}\left\{D(\Phi) H^{+} \mathbf{g}+\Phi H^{+}\left[-D(H) H^{+} \mathbf{g}+H^{+T} D(H)^{T} P_{H}^{\perp} \mathbf{g}+D(\mathbf{g})\right]\right. \\
\left.+\left[-\Phi H^{+} D(H) Y+D(\Phi) Y\right] G^{-}(\mathbf{y}-\zeta)\right\} \\
=-V^{T}\left\{\Phi H^{+}\left[-D(H) \mathbf{b}+H^{+T} D\left(H^{T}\right) P_{H}^{\perp} \mathbf{g}+D(\mathbf{g})\right]+D(\Phi) \mathbf{b}\right\}
\end{aligned}
$$

where $\mathbf{b}=Y G^{-}(\mathbf{y}-\zeta)+H^{+} \mathbf{g}$. 
The matrix $V$ in (2.4) and (2.6) and the matrix $G^{-}$in (2.6) can be computed using the orthogonal decomposition of $G$ given by

$$
G=U\left(\begin{array}{l|l}
R & S \\
\hline 0 & 0
\end{array}\right) P
$$

where $U$ is an orthogonal matrix, $P$ is a permutation matrix and $R$ is a $q \times q$ nonsingular upper triangular matrix. If $U$ is partitioned into

$$
U=(\underbrace{U_{1}}_{q}: U_{2})
$$

then $G^{T} U_{2}=0$ so $V$ can be $U_{2} . V$ can be generated using a sequence of Householder transformations as in [1]. The matrix $G^{-}$can be represented as

$$
P^{T}\left(\begin{array}{l|l}
R^{-1} & 0 \\
\hline 0 & 0
\end{array}\right) U^{T} .
$$

3. Computational procedure. For a fixed value of $\boldsymbol{\alpha}$, the vector $\mathbf{f}(\boldsymbol{\alpha})$ of (2.4) may be constructed as follows:

1) Determine $\Phi(\boldsymbol{\alpha}), H(\boldsymbol{\alpha})$.

2) Determine a complete orthogonal decomposition of $H(\alpha)$ by finding orthogonal matrices $H$ and $Z$ such that

$$
Q H Z=\left(\begin{array}{l|l}
T & 0 \\
\hline 0 & 0
\end{array}\right)
$$

where $T$ is an $r \times r$ nonsingular upper triangular matrix. As in Golub [1] $Q$ and $Z$ may be the products of Householder transformations designed to reduce $H$ to $T$. The matrices $Q$ and $Z$ need not be explicitly formed. Only the information required to generate the Householder transformations need be saved.

3) Form the matrix $C=\Phi Z$ by applying the Householder transformations which form $Z$ to the matrix $\Phi$. The last $n-r$ columns of $C$ form the matrix $G$ in (2.3).

4) Determine the orthogonal matrix $U$ and the permutation matrix $P$ such that

$$
U C\left(\begin{array}{l|l}
I & 0 \\
\hline 0 & \underbrace{P}_{n-r}
\end{array}\right)=\left(\begin{array}{l|l|l}
M & R & S \\
\hline N & \underbrace{0}_{n-r} & 0
\end{array}\right)
$$

where $R$ is a $q \times q$ nonsingular upper triangular matrix. Again $U$ may be the product of Householder transformations and need not be explicitly formed.

5) Compute $\mathbf{d}=\mathrm{H}^{+} \mathbf{g}$ as follows:
(a) Set $\mathbf{a}=Q \mathbf{g}=\left(-\frac{\mathbf{a}_{1}}{-\mathbf{a}_{2}}-\right)^{r}$.
(b) Solve $T \mathbf{c}=\mathbf{a}_{1}$.
(c) Set $\mathbf{d}=Z\left(-\frac{\mathbf{c}}{0}\right)$. 
6) Compute $\mathbf{f}(\boldsymbol{\alpha})$ by setting

$$
\mathbf{p}=U(\mathbf{y}-\Phi \mathbf{d})=\left(\frac{\mathbf{p}_{1}}{\mathbf{p}_{2}}\right)_{m-q}^{q} .
$$

The vector $\mathbf{f}(\boldsymbol{\alpha})$ is contained in $\mathbf{p}_{2}$ and hence $\|\mathbf{f}(\boldsymbol{\alpha})\|_{2}$ is simply $\left\|\mathbf{p}_{2}\right\|_{2}$.

When $B$ of $(2.5)$ is also required, one should continue the procedure as follows:

7) Compute $D(\Phi(\boldsymbol{\alpha})), D(H(\boldsymbol{\alpha}))$, and $D(\mathbf{g})$.

Usually $D(\Phi)$ and $D(H)$ are tensors with many columns that are zero. Golub and Pereyra [2] describe a scheme for storing only the nonzero columns and determining tensor by vector products using this compact storage arrangement.

8) Compute b of (2.6) as follows:

(a) Solve $R \mathbf{e}=\mathbf{p}_{1}$ where $R$ was formed in step 4) and $\mathbf{p}_{1}$ in step 6).

(b) Set $\mathbf{h}=P\left(\begin{array}{l}\mathbf{e} \\ 0\end{array}\right)$.

(c) Set $\mathbf{b}=Z\left(\begin{array}{l}0 \\ \mathbf{h}\end{array}\right)+\mathbf{d}$.

9) Set $R=Q D(H)$ by applying the Householder transformations which form $Q$ to the nonzero columns of the tensor $D(H)$.

10) Form $J=Q\left(D(H) \mathbf{b}-H^{+T}(D H)^{T} P_{H}^{\perp} \mathbf{g}-\mathrm{D}(\mathbf{g})\right)$.

(a) Form $(D H)^{T} P_{H}^{\perp} \mathrm{g}$ by setting

$$
E=R^{T}\left(\begin{array}{c}
0 \\
\mathbf{a}_{2}
\end{array}\right) .
$$

(b) Set $F=Z^{T} E=\left(\frac{F_{1}}{F_{2}}\right)^{r}$

(c) Solve the $k \quad r \times r$ systems

$$
T^{T} G=F_{1} .
$$

(d) Set $J=R \mathbf{b}-\left[\begin{array}{c}G \\ 0\end{array}\right]-Q D(\mathbf{g})=\left(-\frac{J_{1}}{J_{2}}\right)^{r}$.

11) The matrix $B$ is finally obtained as follows:

(a) Solve the $k \quad r \times r$ systems

$$
T K=J_{1}
$$

(b) Set $L=U D(\Phi) \mathbf{b}=\left(\frac{L_{1}}{L_{2}}\right)_{m-q}^{q}$

(c) $B=-\left(N K+L_{2}\right)$.

4. Algorithm implementation and numerical results for linear constraints. Considerable simplifications arise in the aglorithm of $\S 3$ when $H(\boldsymbol{\alpha})$ is a constant matrix. Since this is the case we have actually implemented as a computer code and for which currently we have practical applications we would like to indicate these simplifications. 
Naturally $H(\alpha)$ is not evaluated in 1) each time, since $H$ does not depend upon $\boldsymbol{\alpha}$. For the same reason 2 ) is done once and for all at the beginning of the process. Steps 3)-6) remain the same, while in step 7) $D H(\alpha)$ need not be calculated. Step 8 ) is the same, while 9) is eliminated. In 10) $J$ simply becomes $-Q D(\mathbf{g})$, so parts (a)-(d) are eliminated. Step (11) remains.

The algorithm was implemented in FORTRAN and tested on two examples, in both of which $H$ and $\mathbf{g}$ were constant.

The first problem considered was fitting Gaussians with an expoential background, i.e., the model

$$
a_{1} e^{-\alpha_{1} t}+a_{2} e^{-\alpha_{2}\left(t-\alpha_{3}\right)^{2}}+a_{3} e^{-\alpha_{4}\left(t-\alpha_{5}\right)^{2}}+a_{4} e^{-\alpha_{6}\left(t-\alpha_{7}\right) 2}
$$

is fitted to 65 data points. See [11] for a listing of the data and starting values for $\alpha$. The $a$ 's were constrained to the hyperplanes

$$
\begin{aligned}
a_{1}+2 a_{2}+3 a_{3}+4 a_{4} & =6.27006284, \\
a_{1}+a_{3} & =1.74158318 .
\end{aligned}
$$

This problem was chosen in order to verify the correctness of the formulas and the corresponding code.

With these constraints the solution to the problem coincides with the solution to the unconstrained problem which is available in [2], [11].

With 9 function evaluations, 8 derivative evaluations and 3.23 seconds of computing time on a CDC 6400 computer (Run compiler) the residual $r(\mathbf{a}, \boldsymbol{\alpha})$ was reduced to .04013774 .

The second problem was supplied by Peter Kirkegaard of the Atomic Energy Commission, Risö, Denmark. Kirkegaard and Eldrup [6] had devised a method for solving separable nonlinear least squares problems with linear constraints on the linear variables which arose in the analysis of positron lifetime spectra. Their algorithm used Marquardt's algorithm based on the fact that for a fixed $\boldsymbol{\alpha}$, the optimal arc could be obtained via the symmetric indefinite system

$$
\left[\begin{array}{cc}
\Phi^{T} \Phi & H^{T} \\
H & 0
\end{array}\right]\left[\begin{array}{l}
\mathbf{a} \\
\mathbf{u}
\end{array}\right]=\left[\begin{array}{c}
\Phi^{T} \mathbf{y} \\
\mathbf{g}
\end{array}\right]
$$

Kirkegaard gave the authors an example in which the $\Phi$ matrix was given by

$$
\Phi_{i j}=x_{i}^{1 / 2} \sum_{p=1}^{2} w_{p}\left(z_{i j p}-z_{i+1, j p}-\operatorname{erf}\left\{\left(t_{i}-\alpha_{4}-d_{p}\right) / \sigma_{p}\right\}+\operatorname{erf}\left\{\left(t_{i+1}-\alpha_{4}-d_{p}\right) / \sigma_{p}\right\}\right) .
$$

where

$$
\begin{gathered}
z_{i j p}=e^{-\alpha_{f}\left(t_{i}-\alpha_{4}-d_{p}-1 / 4 \alpha_{i} \sigma_{p}^{2}\right)}\left(1-\operatorname{erf}\left\{\alpha_{j} \sigma_{p} / 2-\left(t_{i}-\alpha_{4}-d_{p}\right) / \sigma_{p}\right\}\right) \quad \text { for } j=1,2,3, \\
\left.\left.z_{i j p}=e^{-\lambda\left(t_{i}-\alpha_{4}-d_{p}-1 / 4 \lambda \sigma_{p}^{2}\right)}\left(1-\operatorname{erf}\left\{\lambda \sigma_{p} / 2-t_{i}-\alpha_{4}-d_{p}\right) / \sigma_{p}\right\}\right)\right),
\end{gathered}
$$

and

$$
\begin{gathered}
w_{1}=6, \quad w_{2}=4, \quad d_{1}=25 / 70, \quad d_{2}=0, \quad \lambda=\frac{.07}{1.7} \\
\sigma_{1}=\frac{.38}{.14[\ln (2)]^{1 / 2}} \quad \text { and } \quad \sigma_{2}=\frac{.485}{.14[\ln (2)]^{1 / 2}}
\end{gathered}
$$


and the values of $x_{i}$ are given in the Table 1 below. Thus $n=4$ and $k=4$. The vector $\mathbf{t}$ was given by

$$
t_{i}=i+121 \text { for } i=1,2, \cdots, 379
$$

and the vector $\mathbf{y}$ was given by

$$
y_{i}=\frac{\left(x_{i}-85\right)}{x_{i}^{1 / 2}} \quad \text { for } i=1,2, \cdots, 379 .
$$

TABLE 1

\begin{tabular}{|c|c|c|c|c|c|c|c|c|c|}
\hline & 101 & 84 & 88 & 99 & 106 & 104 & 100 & 74 & 107 \\
\hline 105 & 109 & 105 & 99 & 90 & 84 & 112 & 141 & 195 & 419 \\
\hline 919 & 2,099 & 4,352 & 7,947 & 12,952 & 18,596 & 24,154 & 27,804 & 29,418 & 28,497 \\
\hline 25,948 & 22,837 & 19,579 & 17,241 & 14,495 & 12,727 & 11,264 & 10,007 & 9,088 & 8,115 \\
\hline 7,473 & 6,642 & 6,131 & 5,626 & 5,192 & 4,691 & 4,324 & 4,136 & 3,677 & 3,461 \\
\hline 3,219 & 2,977 & 2,628 & 2,589 & 2,394 & 2,105 & 1,997 & 1,967 & 1,697 & 1,656 \\
\hline 1,511 & 1,481 & 1,346 & 1,264 & 1,199 & 1,144 & 1,029 & 1,003 & 910 & 891 \\
\hline 847 & 737 & 751 & 695 & 644 & 644 & 600 & 548 & 509 & 465 \\
\hline 447 & 454 & 411 & 371 & 357 & 346 & 320 & 315 & 318 & 292 \\
\hline 252 & 263 & 264 & 252 & 204 & 205 & 204 & 212 & 190 & 195 \\
\hline 198 & 169 & 183 & 171 & 168 & 157 & 159 & 147 & 162 & 152 \\
\hline 153 & 120 & 132 & 157 & 124 & 133 & 106 & 112 & 122 & 129 \\
\hline 104 & 133 & 116 & 102 & 127 & 101 & 105 & 105 & 121 & 86 \\
\hline 84 & 100 & 97 & 99 & 94 & 112 & 89 & 98 & 82 & 87 \\
\hline 82 & 87 & 100 & 95 & 92 & 113 & 88 & 95 & 96 & 104 \\
\hline 102 & 103 & 87 & 80 & 77 & 85 & 84 & 95 & 107 & 91 \\
\hline 80 & 94 & 100 & 88 & 80 & 80 & 88 & 90 & 97 & 84 \\
\hline 75 & 72 & 94 & 77 & 86 & 108 & 63 & 88 & 82 & 106 \\
\hline 77 & 79 & 87 & 79 & 72 & 67 & 90 & 85 & 89 & 91 \\
\hline 86 & 89 & 87 & 102 & 93 & 91 & 97 & 90 & 80 & 87 \\
\hline 82 & 93 & 68 & 82 & 83 & 77 & 76 & 93 & 92 & 90 \\
\hline 68 & 90 & 102 & 77 & 75 & 93 & 87 & 76 & 72 & 78 \\
\hline 84 & 79 & 84 & 93 & 83 & 84 & 89 & 88 & 90 & 84 \\
\hline 91 & 88 & 71 & 93 & 96 & 82 & 89 & 75 & 101 & 70 \\
\hline 78 & 88 & 72 & 70 & 72 & 81 & 83 & 88 & 92 & 85 \\
\hline 91 & 84 & 86 & 82 & 84 & 80 & 88 & 76 & 77 & 97 \\
\hline 85 & 83 & 96 & 92 & 89 & 64 & 91 & 87 & 89 & 85 \\
\hline 79 & 103 & 80 & 100 & 75 & 88 & 79 & 77 & 82 & 81 \\
\hline 81 & 104 & 77 & 98 & 92 & 81 & 78 & 88 & 81 & 91 \\
\hline 78 & 90 & 72 & 95 & 92 & 72 & 77 & 84 & 78 & 88 \\
\hline 83 & 80 & 76 & 91 & 79 & 83 & 80 & 76 & 75 & 81 \\
\hline 105 & 80 & 94 & 77 & 102 & 88 & 92 & 79 & 93 & 93 \\
\hline 82 & 82 & 82 & 87 & 86 & 99 & 78 & 82 & 77 & 90 \\
\hline 83 & 104 & 85 & 95 & 89 & 94 & 70 & 83 & 84 & 92 \\
\hline 89 & 99 & 85 & 97 & 99 & 86 & 76 & 98 & 84 & 90 \\
\hline 86 & 69 & 79 & 98 & 82 & 73 & 78 & 79 & 75 & 78 \\
\hline 95 & 76 & 75 & 97 & 77 & 103 & 88 & 86 & 90 & 87 \\
\hline 93 & 76 & 72 & 91 & 85 & 98 & 107 & 99 & 81 & 71 \\
\hline 90 & 82 & 78 & 80 & 63 & 91 & 93 & 89 & 75 & 80 \\
\hline 72 & 83 & 82 & 86 & 103 & 90 & 83 & 90 & 96 & 80 \\
\hline
\end{tabular}

(x values) 
There was only 1 linear constraint for this particular problem:

$$
.54 a_{1}+.54 a_{2}+.46 a_{3}+.54 a_{4}=0 .
$$

Initially $\boldsymbol{\alpha}$ was $(.54, .2, .07,127.4)^{T}$ which gave a residual of $1,353.036$. The residuals at successive iterations were

$$
\begin{aligned}
& 426.9649 \\
& 359.8339 \\
& 359.1253 \\
& 359.0751 \\
& 335.0720
\end{aligned}
$$

Initially $\nu_{j}$ in (2.5) was set to $\left(\|B\|_{2}^{2} /(m \cdot k)\right)$. On successive iterations $\nu$ was half of its previous value. The final $\alpha$ was $(.53777671, .211172, .073373458$, $126.92371)^{T}$ while the final a was $(32,783.984,52,140.2229,108,630.17$, $7,612.5942)^{T}$.

Acknowledgment. The authors would like to thank Prof. Gene Golub, Stanford University, for his support and hospitality.

\section{REFERENCES}

[1] G. H. Golub, Matrix decompositions and statistical calculations, Statistical Computation, R. C. Milton and J. A. Nelder, eds., Academic Press, New York, 1970.

[2] G. H. GOlub AND V. PEREYRA, The differentiation of pseudoinverses and nonlinear least squares problems whose variables separate, this Journal, 10, (1973), pp. 413-432.

[3] - Differentiation of pseudoinverses, separable nonlinear least squares problems, and other tales, Proc. MRC Seminar on Generalized Inverses and their applications, Z. Nashed, ed., Madison, Wisconsin (October 1973), 1976, pp. 303-324.

[4] L. S. Jennings AND M. R. Osborne, Applications of orthogonal matrix transformations to the solution of systems of linear and nonlinear equations, Tech. Rep. 73, Computer Centre, Australian National Univ., Canberra, 1970.

[5] L. KAUFMAN, A variable projection method for solving separable nonlinear least squares problems, BIT, 15 (1975), pp. 49-57.

[6] P. KIRKegAard AND M. EldRup, Positronfit: A versatile program for analyzing positron lifetime spectra, Computer Physics Comm., 3 (1972), pp. 240-255.

[7] F. KROGH, Efficient implementation of a variable projection algorithm for nonlinear least squares problems, Comm. ACM, 17 (1974), pp. 167-169.

[8] A. Perez AND H. D. Scolnik. Derivatives of pseudoinverses and constrained nonlinear regression problems, Numer. Math., to appear.

[9] R. C. RAO AND S. K. MitrA, Generalized Inverses of Matrices and its Applications, John Wiley, New York, 1971.

[10] A. RuHE AND P. A. Wedin, Algorithms for separable nonlinear least squares problems, Stanford University Computer Science Rep. STAN-CS-74-434, Stanford, CA, 1974.

[11] M. R. OsBORNE, Some aspects of nonlinear least squares calculations. Numerical Methods for Nonlinear Optimization, F. A. Lootsma ed., Academic Press, London, 1972. 